Rev. Chil. Pediatr. 66 (6); 341-344, 1995

\title{
Estadística en el Programa Epiinfo 6.0
}

\author{
Gastón Duffau T. ${ }^{1}$
}

\section{Critical review of some aspects of Epiinfo 6.0's statistical programe}

\begin{abstract}
A critical review of Epinfo 6.0's statistical programme was done and some problems were observed, mainly when oderating STALCAIC and EPITABLE routines for chi square. Yales's correction, odds ratios with zeros, stratified analysis, averages comparison, interrater agreement and vcccines effectivity cohort study calculalions. When chi square analysis is applied to a $2 \times 3$ data table using STATCALC the programe gives results on stalistics and their asscxiated " $p$ " value even if expected volue is less than one, which is not possible when EPITABLE is used. When EPITABIE is appiad to $2 \times 2$ data lables it calivilates chi square volues with Yates correction only if expected vaive is less than 5. STATCAIC de:ermines or does nct odds ratios with zeros, depending on their place in the data tables. Even more, it accepts and makes calcularions with two zeros if these are placed in the main diagonal line and in stratitied analysis- it does not inciude the Mantel Haenszel test for homogeneity. EPITABLE does not offer the slandard error of kappr when used to delermine interobserver agreemert, and it does not determine weighted koppo value when this should be done. When comporing means values through varience anaysis for two groups. EPITABIE does ror warn abou! differences in voriances if inere are more than 30 cases in each group. in immunization efficoor estimates by cohort studies the "Help" oplion offers a wrong solurion.
\end{abstract}

(Koy words: biometry, compurer assisted. epidemiological methods, scalistics.)

EPIINFO es la denominación de un conjunto lógico definido como "programa procesador de palabras, base de datos y estadístico para Salud Pública". Tiene un grado de difusión elevado por ser de gran utilidad, estar avalado por la Organización Mundial de la Salud así como por el Centro de Control de Enfermedades de Atlanta (EUA) y porque se obtiene en las oficinas respectivas sin costos para el interesado. Este tipo de instrumentos de trabajo va asociado a ideas de perfección y de cierta infalibilidad en todo desempeño, lo que puede inducir a su utilización sin prevención alguna. El objetivo de este estudio fue verificar hasta qué punto el programa mencionado se ajusta, en la parte de análisis estadístico de resultados, a ciertas normas generalmente aceptadas $y$, al mismo tiempo, establecer los aspectos en los cuales muchos usuarios pudieran requerir de cierto cuidado.

1. Universidad de Chile, Facultad de Medicina. Departamento de Pediatria, Campus Norte.

Con financiamiento de Proyecto Fondecyt $N^{\circ} 1950656$.

\section{Material y Método}

Se dispuso del programa EPINFO versión 6.0 de abril de 1994 y se revisaron, of de problemas, parte de los subprogramas estadísticos STATCALC Y EPJTABLE, escogiendo en forma arbitratia dentro de ellos tas rutinas probablemente más empleadas por clínicos, con la intención de hacer al lector algunas advertencias que pudieran ser útiles al analizar con EPIINFO los datos de una investigación.

\section{Resultados y Comentario}

STATCALC, en tabla de $2 \times 2$, indica ventaja o razón de productos cruzados ("odds ratio, OR" $)^{1.2}$ y entrega los resultados de la prueba de Ji cuadrado con corrección de Yates ${ }^{3}$ y sin ella. Si hay una frecuencia esperada menor de 5 , recomienda efectuar la prueba de Fisher-Irwin de probabilidades exactas ${ }^{4}$, pero cuando la tabla es $2 \times 3$ o más grande, el usuario puede encontrar que en algunas tablas donde hay cifras esperadas menores de 5 el progrania advierte sobre este hecho e indica que la prueba de $\mathbf{J}$ cuadrado no es válida, pero en otras tablas no 
ocurrirá esto y se entregará el resultado de la prueba. Así por ejemplo, en la tabla siguiente:

$\begin{array}{rr}0 & 1 \\ 2 & 1 \\ 9 & 7 \\ 8 & 9 \\ 14 & 0\end{array}$

se encuentran cinco celdas con valores esperados inferiores a 5 y el programa no calcula $\mathrm{Ji}$ cuadrado, expresando que "hay un valor esperado menor que 5 en una celda". Sin embargo, en esta otra:

$\begin{array}{rr}0 & 1 \\ 2 & 1 \\ 9 & 7 \\ 8 & 9 \\ 15 & 0\end{array}$

hay 4 celdas con cifras esperadas inferiores a 5 y pese a ello se indica que $J i$ cuadrado es 12,94 con 4 grados de libertad y $p=0,011$. Esta misma tabla analizada por EPITABLE señala, en cambio, "invalid input" porque ningún valor esperado puede ser inferior a 1 .

EPITABLE en tabla de $2 \times 2$ entrega los resultados sin corrección por continuidad, excepto cuando la cifra esperada en una celda es inferior a 5 (ver opción "Help" (Ayuda)), lo que resulta sorprendente porque la corrección de Yates no persigue resolver este problema. Si hay una cifra esperada menor que la unidad, no efectúa la prueba y señala "invalid input". Para tablas tnayores muestra el porcentaje de valores esperados inferiores a 5 y calcula Ji cuadrado. Ante cifras esperadas inferiores a 1 , como se explic 6 , no realiza la prueba.

La corrección de Yates se considera importante en tabla de $2 \times 2$, al aproximar la distribución de Ji cuadrado a la normals. Una situación similar se da con la distribución binomial. Es cierto que la corrección hace la prueba más conservadora y también to es que no todos la apli. $\mathrm{can}^{6.7}$, pero lo claro es que EPINFO 6.0 la incluye o no según el programa que esté corriendo. Señalaremos que para la ejecución de la prueba de Ji cuadrado es generalmente aceptado que no debe haber ningún valor esperado menor que 1 ni más de $20 \%$ de celdas con valor esperado inferior a 5 (entonces, en tabla de $2 \times 2$ no puede haber ninguna). La solución aquí es reagrupar categorías vecinas ${ }^{1-5}$.

Las ventajas ("OR") son calculadas en STATCALC y se entregan sus límites de confianza según Cornfield ${ }^{1}$, aunque se pueden obtener valores exactos. Cuando en la tabla hay un cero, el programa entrega resultado de ventajas o no lo hace (declarando OR "indefinido") dependiendo de la posición del cero. Así es como, si éste está ubicado en la celda "a" o "d" muestra valores, cosa que no hace si el cero se encuentra en las celdas "b" $o$ "c". Es más, si se ponen dos ceros uno en "a" y el otro en "d", también da un resultado. Esto es raro si consideramos que para el cálculo de ventajas en presencia de un cero se han propuesto soluciones que no justificarían expresarlo como "indefinido" 1,8 . Por otra parte, el problema persiste independientemente de la localización del cero, ya que "OR" resultaría un valor incalculable $(a \times d /$ b x 0 o a $\times d / 0 \times c)$ o cero $(a \times 0 / b \times c \circ 0 \times d / b$ $x$ c) y por último, con dos ceros se dan situaciones que no siempre tienen sentido.

Cuando se desea efectuar un análisis estratificado de datos, STATCALC determina la totalidad de las etapas pero no incluye la muy importante prueba de homogeneidad de Mantel y Haenszell.

EPITABLE pide -para el cálculo de intervalo de confianza $(95 \%)$ de un promedio- el tamano de la población de la que se extrajo la muestra, además del tamaño muestral, el promedio y la desviación estándar. Esto es bastante inusual ya que raramente se dispone del tamaño de la poblacion. De todos modos, al faltar el dato el programa supone una población de 99.999 .999 efectivos. La idea es que si la muestra tiene un tamaño más cercano al de la poblacion, el intervalo de confianza es más estrecho que si ocurre lo opuesto.

Para el análisis de concordancia en la evaluación por dos observadores, el programa calcula el estadístico kappa corregido (para efecto del azar) y el error estándar para la eventualidad que kappa $=0^{9}$. Además señala el valor de " $p$ " asociado. Estos cálculos los efectúa de la misma manera en tabla de 2 x 2 y otras mayores. De hecho, no toma en consideración la importancia del grado de discordancia, que tiene sólo una expresion si la tabla es $2 \times 2$, puesto que dos de las cuatro celdas corresponden a acuerdo entre los observadores y las restantes dos a desacuer- 
do. Sin embargo, en una tabla mayor, existen grados de desacuerdo que el programa no contempla. De hecho, en una tabla como la que se muestra a continuación :

$\begin{array}{rrrr}20 & 5 & 0 & 0 \\ 2 & 30 & 3 & 0 \\ 0 & 3 & 40 & 2 \\ 0 & 0 & 5 & 10\end{array}$

kappa corregido $=0,76$

se obtiene un índice kappa prácticamente igual que en la siguiente, pese a que la discordancia, siendo igual el número de casos, es absolutamente diferente:

$\begin{array}{rrrr}20 & 0 & 0 & 10 \\ 0 & 30 & 0 & 0 \\ 0 & 0 & 40 & 0 \\ 10 & 0 & 0 & 10\end{array}$

kappa corregido $=0,77$

Para resolver esto se debe calcular el estadístico kappa ponderado ${ }^{9}$, que reflejará mejor la situación, ya que en el primer caso señalará un valor de 0,83 y en el segundo s6lo 0,56. Por otra parte, tampoco se entrega el error estándar de kappa encontrado, lo que hace engorroso para el usuario el comparar dos de estos estadísticos, debiendo efectuar el cálculo por sí mismo. Recordaremos que el error estándar que entrega sólo permite decir cuán diferente de cero es el valor entregado de kappa.

Al comparar una distribución determinada con otra teórica por medio de Ji cuadrado en la opción "bondad del ajuste" ("Goodness of fit"), el número de grados de libertad no aparece correctamente si hay cifras esperadas inferiores a 5 y - si se coloca un cero- simplemente el programa se devuelve al comienzo de EPIINFO 6.0 .

Si se desea comparar promedios no aparece otra posibilidad que el análisis de varianza que - "Help" explica- es, para dos muestras, una prueba equivalente a " $t$ " de Student $t^{10}$ y que para poder efectuarla en propiedad se requiere que las varianzas no sean estadísticamente diferentes. Si lo son, empleará el procedimiento de Satterthwaite ${ }^{11}$ que corrige los grados de libertad, haciendo más exigente el método, esto es, requiriendo un valor de " $t$ " mayor para declarar diferente los promedios. Sobre esto hay que aclarar que cuando se dan las circunstancias, aparece en la pantalla que las varianzas son di- ferentes, se indica el "p" asociado y luego viene la comparación de promedios. Queda señalado, además, que se empleará el método đe Satterthwaite. Sin embargo, aunque las varianzas sean diferentes, cosa comprobable con la rutina "comparación de varianzas", si los grupos en estudio tienen 30 o más casos, no aparece ninguna de las indicaciones señaladas y sólo se destaca la comparación de promedios. Esto no está bien, ya que dos grupos pueden ser diferentes por sus varianzas, sus promedios o ambos ${ }^{7}$, lo que nos llevaría, entonces, al punto de partida correcto, es decir, a la rutina ofrecida como comparación de varianzas, prueba " $\mathrm{F} " 10$. Si la razón varianza mayor /varianza menor indica que éstas son significativamente distintas, también lo son los grupos que se están comparando. Si se insiste en contrastar los promedios, se requerirá una corrección de los grados en libertad, que pudiera ser la propuesta o bien aquélla sugerida por Welch ${ }^{12}$.

EPITABLE puede proporcionar el intervalo de confianza de una proporción, pero es necesario tener presente que cuando ésta es muy pequeña, cercana a cero, el tiempo que demora el programa en dar una respuesta se va dilatando progresivamente.

En el estudio de eficacia de vacunas por medio de cohortes se calcula el riesgo relativo' (RR), pero también se incluye $O R$, lo que no corresponde, puesto que $O R$ representa una aproximación al $R R$ cuando la situación estudiada tiene poca frecuencia y se trata de un diseño caso-control, precisamente porque una investigación de cohortes no ha sido posible.

La eficacia de una vacuna aparece definida como (l-RR) x 100 o bien, para estudio casocontrol, (1-OR) x 100.

En cohortes, "Help" presenta :

\section{Enfermedad}

$\begin{array}{ccccc}\text { Vacunados } & \text { (sí) } & \text { (si) } & \text { (no) } & \\ & \text { (no) } & c & b & a+b \\ & & a+c & b+d & c+d\end{array}$

En donde, el riesgo de enfermar en los sujetos vacunados es $a /(a+b)$ y en los no vacunados $c /(c+d)$. El RR será la razón $a /(a+b)$ cuyo valor $\mathrm{b} /(\mathrm{c}+\mathrm{d})$ inferior a uno, se utiliza justamente para determinar eficacia de vacunas en la fórmula 
señalada más arriba. Ahora bien, en "Help" se propone calcular RR de la siguiente manera: a $x$ $(b+d)$ lo que es b $x(a+c)$, claramente un error. Como sea, si se construye un ejemplo, el programa genera un resultado correcto pero la instrucción para hacerlo da un valor diferente y equivocado.

\section{Conclusiones}

En lo principal, al analizar una tabla de $2 \times 3$ - mayor con Ji cuadrado por STATCALC se obtienen resultados del estadístico y de " $p$ " asociado a éste aunque el valor esperado sea inferior a uno. Por EPITABLE ello no es posible.

EPITABLE, en tabla de $2 \times 2$, calcula Ji cuadrado con corrección de Yates sólo si hay un valor esperado $<5$.

STATCALC determina "OR" con ceros, o no lo hace, dependiendo de la ubicación de éstos en la tabla. Incluso acepta y calcula con 2 ceros siempre que estén en la diagonal principal. En análisis estratificado, no incluye la prueba de homogeneidad de Mantel y Haenszel.

EPITABLE, en estudio de concordancia, no proporciona el error estándar del índice kappa calculado y, cuando corresponde, no determina kappa ponderado. En comparación de promedios por análisis de varianza (2 grupos) -si éstas difieren- lo advierte, excepto si hay más de 30 casos en cada grupo. En el estudjo de eficacia de vacunas por medio de cohortes, la opción "ayuda" propone una solución equivocada.

\section{Resumen}

En un estudio crítico de las rutinas estadísticas del programa EPIINFO versión 6.0, $1994 \mathrm{se}$ advierten posibles problemas para el usuario en la prueba de Ji cuadrado, ya que en tablas de
$2 \times 3$ o mayores -con STATCALC- se obtienen resultados estadisticos y de " $\mathrm{p}$ " asociado aunque el valor esperado sea menor que uno, lo que no es posible conseguir con EPITABEE. También se detectan fallas en la corrección de Yates, cálculo de ventajas ("odds ratios") con ceros, análisis estratificado, comparación de dos promedios, estudio de concordancia y en evaluación de vacunas por medio de cohortes.

(Palabras clave: biometría, estadística, métodos epidemiológicos, programas computadorizados.)

\section{Referencias}

1. Fleiss J: Satatistical methods for rates and proportions. 2nd. edition, New York: John Wiley \& Sons, 1980: 56-82.

2. Matihens $D$, Farewell $V$ : Estadistica médica. Aplicación e interpretacion. Barcelona: Salvar Editores, S.A., 1988: 11-66

3. Siegel $S$ : Nonparametric statistics for the behavioral sciences. New York: McGraw-Hill Book Co., 1956: $95-110$.

4. Saunders BD, Trapp R: Bioestadística médica. México DF: Manual Moderno, S.A., 1993: 173-180.

5. Ingelfinger $J$, Mosteller $F$, Thibodeau $L$, Ware $J$. Biostatistics in clinical medicine. 2nd edition. New York: Macmillan Publishing Co., 1987: 168-180.

6. Colton T: Estadistica en ruedicina. Barcelona: Salvat Editores, S.A., 1979: 183-190.

7. Sclwarfz $D$ : Métodos estadísticos para médicos y biólogos. Barcelona: Herder 1985: 87-105.

8. Valenzuela $C$ : Dos soluciones para la estimación de odds ratios con ceros. Rev Med Chil 1993: 121-: 1441-1444

9. Kramer $M$, Feinstein A: Clinical biostatistics. LIV. The biostalistics of concordance. Clin Pharmacol Ther 1981; 29: 111-123.

10. Lamotte M: Estadistica biológica. Barcelona: TorayMasson S.A. 1965: 92-100.

11. Steel R, Torrie J: Bioestadística: Principios y procedimientos. Madrid: McGraw-Hill, 1990: 102-105

12. Lentuer $C$; Introduction to statistics in: Scientific tables, 8th edition, Switzerland: Ciba-Geigy Lud. 1982: 209. 